\title{
Infrared Optical Constants and Computational Studies of Neat Liquid $n$-Butylethylether
}

\author{
K. B. Beć and J. P. Hawranek \\ Faculty of Chemistry, University of Wrocław, F. Joliot-Curie 14, 50-383 Wrocław, Poland \\ Correspondence should be addressed to K. B. Beć; krzysztof.bec@chem.uni.wroc.pl
}

Received 22 June 2012; Accepted 14 August 2012

Academic Editor: Renata Diniz

Copyright (C) 2013 K. B. Beć and J. P. Hawranek. This is an open access article distributed under the Creative Commons Attribution License, which permits unrestricted use, distribution, and reproduction in any medium, provided the original work is properly cited.

\begin{abstract}
Aliphatic ethers are of interest to researchers due to their wide application in the fuel, chemical, and pharmaceutical industry. In this paper we studied vibrational properties of neat liquid $n$-butylethylether (NBEE), including the determination of complex refractive index in the NIR and MIR range $\left(11700-560 \mathrm{~cm}^{-1}\right)$. The high absorption of neat liquid in the MIR range required the use of thin-film transmission recordings. The spectra analysis was based on conformational analysis and anharmonic calculations on B2PLYP/N07D level of theory. Final band assignments procedure was based on potential energy distributions. The theoretical investigation revealed that although 26 conformers of NBEE can be expected to exist in the liquid phase at $298 \mathrm{~K}$, only few of them are essential for the forming of the spectrum. This study is important for the proper understanding of vibrational properties of other aliphatic ethers.
\end{abstract}

\section{Introduction}

Aliphatic ethers have widespread industrial applications, ranging from solvents [1] and catalysts components [2] to fuel additives [3]. The utilization of $n$-butylethylether (NBEE) in fuel industry also lead to an increasing interest in the environmental studies of $n$-butylethylether in the last years [4-6]. Surprisingly the infrared properties of liquid $n$ butylethylether were never deeply investigated. In this paper, we aimed to obtain an insight into vibrational properties of the studied ether through a broad spectral range complex refractive index measurements and a detailed vibrational analysis, with the hope to fully understand factors determining the vibrational spectra of NBEE in the neat liquid phase. The determination of these data required the use of thin-film quantitative techniques in the MIR region due to strong absorption in the studied region. Experimental data are supported by a conformational analysis and subsequently a detailed anharmonic vibrational analysis. The final band assignments procedure is based on modelled on B2PLYP/N07D level vibrational spectra of all resolved NBEE conformers.
The results of studies reported here are indispensable for the determination of high frequency dielectric properties of $n$-butylethylether in the liquid phase.

\section{Experimental}

The NBEE sample was of the highest purity available from Aldrich, additionally dried and stored over molecular sieves under nitrogen.

NIR and MIR spectra were recorded on a Nicolett Magna 860 FT-IR/Raman spectrometer. To minimize the effect of possible drift, the empty chamber was measured as a reference spectrum before and after the sample measurement. All measurements were carried out at the controlled temperature $298 \mathrm{~K}$.

Due to strong absorption bands, the MIR spectra were recorded in thin-film cells assembled for the purpose of this work from $\mathrm{KBr}$ windows polished to high flatness, monitored in sodium light on a glass optical flat. The spacers were prepared out of aluminum foils. The geometrical parameters of the cell cavity were determined by fitting the experimental interference spectrum of the empty cell with the theoretical 
ones, using the procedure based on the earlier derived algorithm. Several $\mathrm{KBr}$ cells were used with thickness ranging from 4 to $8 \mu \mathrm{m}$. The resolution was set at $0.5 \mathrm{~cm}^{-1}$ and a scan number of 512 was chosen to ensure good signal to noise ratio.

Spectra in the NIR range were measured on the same instrument. $\mathrm{A} \mathrm{CaF}_{2}$ beamsplitter with the MCT-A detector (in the region $11700-8000 \mathrm{~cm}^{-1}$ ) and DTGS detector (in the $8000-4000 \mathrm{~cm}^{-1}$ region). The resolution was maintained on a $0.5 \mathrm{~cm}^{-1}$ level. Thermostated quartz cells (Hellma) of 5 and $10 \mathrm{~mm}$ thickness were used in the upper NIR region $\left(11700-8000 \mathrm{~cm}^{-1}\right)$; in the lower region cells of 0.1 and $0.5 \mathrm{~mm}$ thickness were used. The absorbance spectrum of the liquid was obtained by subtraction of the empty-cell spectrum from the spectrum of the cell filled with the liquid. Subsequently, the absorbance spectrum was recalculated to yield the spectrum of the absorption index $k(v)$.

FT-Raman spectra were measured on a Nicolet Magna 860 FTIR spectrometer interfaced with a FT-Raman accessory. The samples were illuminated by a $\mathrm{Nd}: \mathrm{YVO}_{4}$ laser line at $1.064 \mathrm{~nm}$ with a power of $0.2-0.3 \mathrm{~W}$. $\mathrm{A} \mathrm{CaF}_{2}$ beamsplitter was used in combination with an InGaAs detector. The interferograms were averaged over 1024 scans. The resolution of the spectra was $2 \mathrm{~cm}^{-1}$.

\section{Data Processing}

From the transmission spectra in the entire measured region, the spectrum of both components of the complex refractive index:

$$
\widehat{n}(v)=n(v)+i k(v)
$$

where $i=\sqrt{-1}$ and $v$ denotes wavenumbers $\left(\mathrm{cm}^{-1}\right)$ throughout this work.

The real and imaginary parts of the complex refractive index are often called customarily optical constants, although they depend on frequency, temperature, and pressure for a given liquid. These dimensionless spectra fully describe optical properties of any isotropic system. Usually the interest of spectroscopists is focused on the spectrum of the absorption index, $k(v)$. Unfortunately, it is not easy to obtain the optical constants; what is more, an exact procedure usually requires a simultaneous determination of both components. Various methods for determination of optical constants were reviewed by Bertie [7]. In our studies, we use the transmission method $[8,9]$ with later enhancements $[10,11]$. The method is based on transmission measurements in the MIR and NIR ranges and refractive indices determined in the visible. A serious difficulty in this method is connected with measurements of spectra in MIR region. Due to strong absorption in this range, a very thin layers have to be used for quantitative measurements and this causes serious dispersion distorsion of the measured spectra [8]. From these data, both component spectra of the complex refractive index are obtained by the use of the procedure combining an iterative correction of the dispersion distortion [8] with the

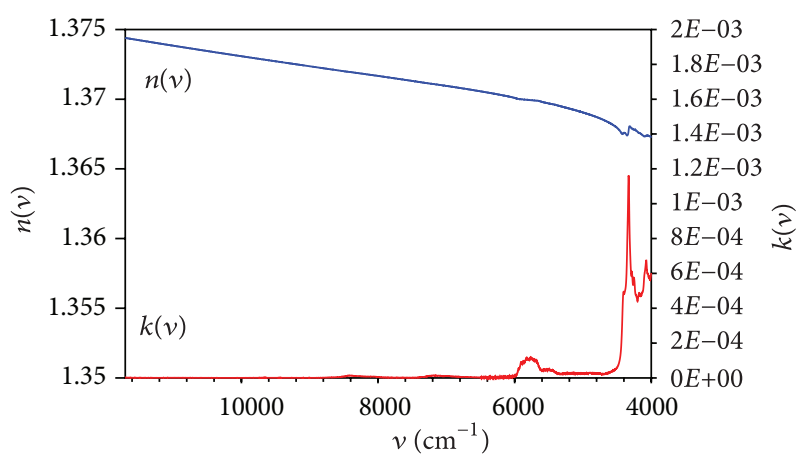

FIGURE 1: Spectrum of the complex refractive index of NBEE in the NIR range $\left(11700-4000 \mathrm{~cm}^{-1}\right)$.

modified Kramers-Krönig procedure [11]. The spectrum was processed as a whole from 11700 to $560 \mathrm{~cm}^{-1}$.

The spectra of the complex refractive index are required for the determination of high frequency dielectric properties of studied liquid, which are in progress. Moreover, the accurate determination of the absorptive properties of the liquid can be based only on the absorption index spectrum $k(v)$ because of the discussed above strong distortion of thin layers transmission spectra [8].

\section{Results and Discussion}

4.1. Complex Refractive Index in the NIR Range (11700$\left.4000 \mathrm{~cm}^{-1}\right)$. The complete spectrum of the complex refractive index in the measured range $\left(11700-4000 \mathrm{~cm}^{-1}\right)$, covering the first and second overtone regions, is presented in Figure 1.

As can be seen for the studied ether, the NIR bands are very weak except for bands in the region $6000-4000 \mathrm{~cm}^{-1}$. In general, the range between $6000-5600 \mathrm{~cm}^{-1}$ reflects well the doubled $3000-2800 \mathrm{~cm}^{-1}$ region. The analysis of the overtones for the studied molecule was not the aim of this work and will not be presented here. However, the $\widehat{n}(v)$ spectrum had to be determined in the NIR region, since its knowledge is essential for an accurate determination of the $\widehat{n}(v)$ spectrum in the MIR region [11].

4.2. Complex Refractive Index in the MIR Range (4000$\left.560 \mathrm{~cm}^{-1}\right)$. Spectra of complex refractive index of NBEE separated into upper MIR region (4000-2000 $\mathrm{cm}^{-1}$ ) and lower MIR region $\left(2000-560 \mathrm{~cm}^{-1}\right.$ ) can be found in Figures 2 and 3, respectively. The detailed discussion of the MIR range will be presented later in this paper. Raman spectrum is presented in Figure 4.

4.3. Anharmonic Vibrational Analysis. In our theoretical research, we possibly seek most accurate reproduction of experimental spectra, as it is a step needed for the following studies of dielectric properties of examined liquids. To achieve that in case of aliphatic ethers, the analysis of theoretical spectra of various conformers is needed [12]. 


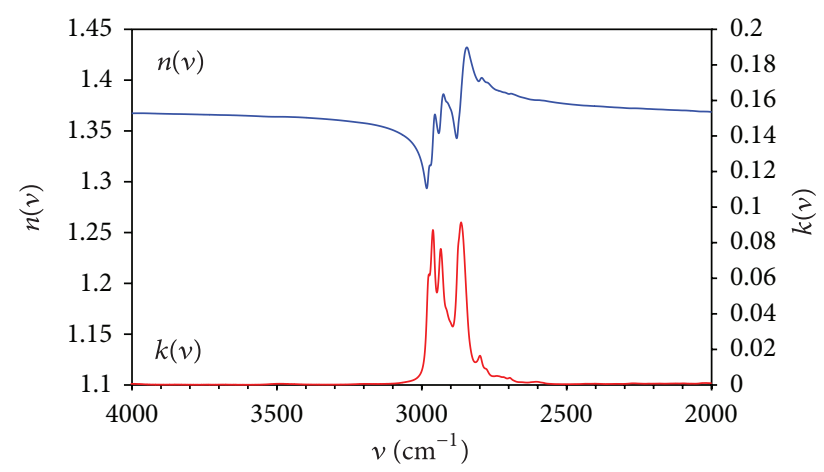

FIGURE 2: Spectrum of the complex refractive index of NBEE in the upper MIR range $\left(4000-2000 \mathrm{~cm}^{-1}\right)$.

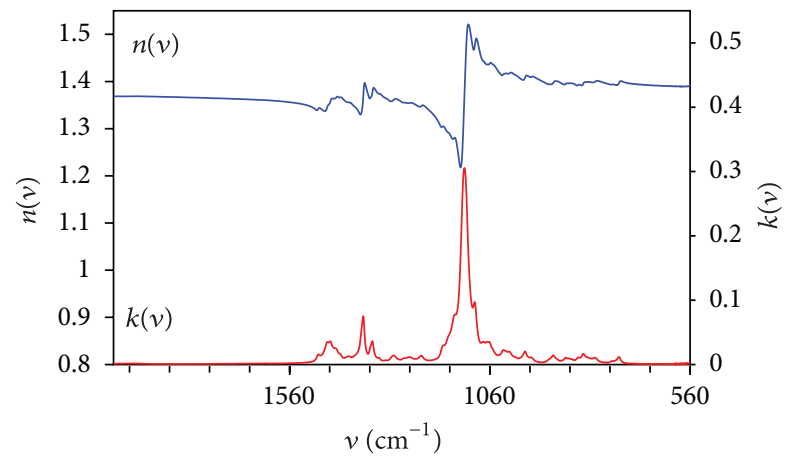

FIGURE 3: Spectrum of the complex refractive index of NBEE in the lower MIR range $\left(2000-560 \mathrm{~cm}^{-1}\right)$.

Also the use of anharmonic calculations on relatively high level is inevitable. In our previous studies [13], we found the hybrid DFT functional B2PLYP with the N07D basis set as exeptionally valuable, considering the accuracy of calculated frequencies and computational cost. Our findings confirm the reported in the literature advantages of discussed computational method [14].

We based the conformational analysis of NBEE on results of previous studies of $n$-butylmethylether (NBME) [15]. We combined 11 stable conformations of the butyl chain found earlier [15] with three possible conformations of the ethyl chain. As a result we obtained 33 initial conformations of NBEE. Eventually 7 of them were found redundant and excluded due to symmetry operations-resulting in a total of $26 \mathrm{NBEE}$ conformations, which were subsequently optimized on a B2PLYP/N07D level of theory.

We assumed the following name scheme for NBEE conformers: $x y z a$, where $x$ denotes conformation ("trans", "gauche+" or "gauche-"; $\mathrm{T}_{1} \mathrm{G}^{+}$or $\mathrm{G}^{-}$, resp.) on a $\mathrm{O}-\mathrm{C}_{\alpha}$ bond, $y$ on a $\mathrm{C}_{\alpha}-\mathrm{C}_{\beta}$ bond, and $z$ on a $\mathrm{C}_{\beta}-\mathrm{C}_{\gamma}$ bond in the butyl chain, and similarly $a$ denotes the conformation on $\mathrm{C}_{\alpha}^{\prime}-\mathrm{O}$ bond in the ethyl chain. This corresponds to our notation scheme used earlier.

The detailed data on resulting NBEE conformer population is presented in Table 1. The "TG-T T" conformer is clearly dominant, with a $39 \%$ concentration, followed by "TG+G+ T" and "TTT T" conformers both with 13\%

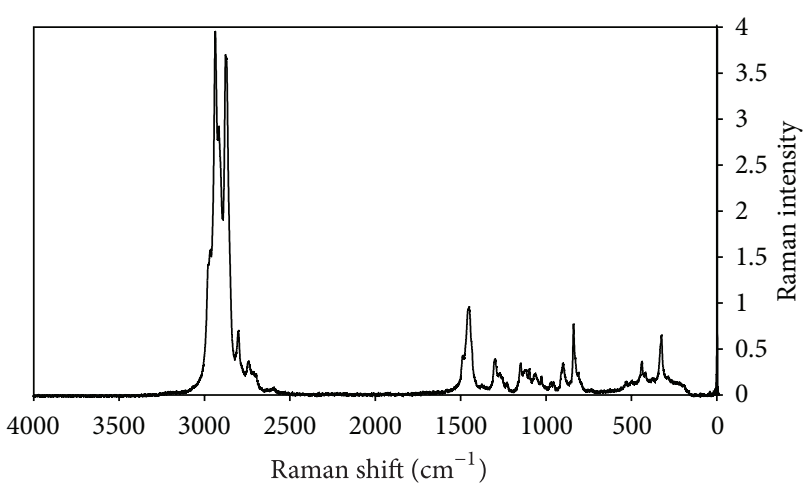

FIgURE 4: Raman spectrum of liquid NBEE.

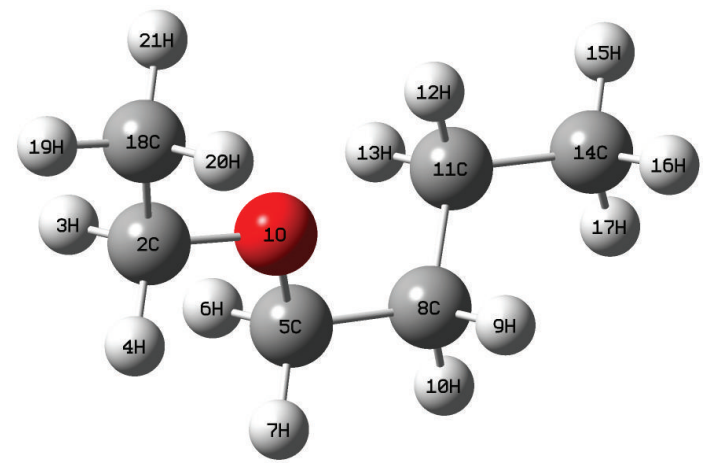

FIGURE 5: Atom numbering in the NBEE molecule.

contributions. As it can be noticed, only few conformers have concentrations high enough to manifest itself in the experimental vibrational spectrum. Nevertheless, the modelled theoretical spectrum involves all investigated forms. The calculated abundances are Boltzmann based.

4.4. Modelled Spectra of NBEE Conformers. Gaussian 09 [16] was used to calculate the spectra of each conformer. The potential energy distribution was calculated using the gar2ped package [17], and the normal coordinates were constructed in accordance to Pulay et al. [18]. The selection of modes was based on the dominant PED values. The definitions of internal coordinates for NBEE molecule are shown in Table 2. The atom numbering in the NBEE molecule can be found in Figure 5.

Table 3 presents PED values for the most abundant (39\%) DNBE conformer("TG-T T"). Due to the high volume of data, PED tables for other conformers are not shown, but are available from authors upon request. The theoretical spectra of 8 most abundant NBEE conformers are presented in Figure 6.

4.5. Simulation of Experimental Spectra. The final calculated spectrum of $n$-butylethylether was obtained as a linear combination of calculated spectra of all conformers with regard to their calculated abundances. The number of conformers taken into account is 26 ; however, as can be concluded from 
TABLE 1: Relative B2PLYP/N07D energies $\Delta E$, relative B2PLYP/N07D energies with zero-point correction $\Delta E_{\mathrm{ZPE}}$, relative free Gibbs energies $(298.15 \mathrm{~K})$, and abundances of NBEE conformers.

\begin{tabular}{|c|c|c|c|c|c|}
\hline Conformer & $\Delta E(\mathrm{kcal} / \mathrm{mol})$ & $\Delta E_{\mathrm{ZPE}}(\mathrm{kcal} / \mathrm{mol})$ & $\Delta G(\mathrm{kcal} / \mathrm{mol})$ & Symmetry & Rel. abundance (\%) \\
\hline TG-T T & 0 & 0 & 0 & $\mathrm{C}_{1}$ & 38.8 \\
\hline $\mathrm{TG}+\mathrm{G}+\mathrm{T}$ & 0.80 & 0.70 & 0.63 & $\mathrm{C}_{1}$ & 13.4 \\
\hline TTT T & 0.08 & 0.29 & 0.23 & $\mathrm{C}_{\mathrm{s}}$ & 13.1 \\
\hline TTG - T & 1.04 & 1.16 & 1.04 & $\mathrm{C}_{1}$ & 6.7 \\
\hline $\mathrm{TG}-\mathrm{G}+\mathrm{T}$ & 1.72 & 1.45 & 1.33 & $\mathrm{C}_{1}$ & 4.1 \\
\hline TG-T G+ & 1.60 & 1.57 & 1.38 & $\mathrm{C}_{1}$ & 3.8 \\
\hline TG-T G- & 1.61 & 1.57 & 1.41 & $\mathrm{C}_{1}$ & 3.6 \\
\hline $\mathrm{G}+\mathrm{G}+\mathrm{T} \mathrm{G}-$ & 1.70 & 1.62 & 1.42 & $\mathrm{C}_{1}$ & 3.5 \\
\hline G+TT T & 1.68 & 1.83 & 1.56 & $\mathrm{C}_{1}$ & 2.8 \\
\hline G+TT G- & 1.68 & 1.87 & 1.68 & $\mathrm{C}_{1}$ & 2.3 \\
\hline $\mathrm{TG}+\mathrm{G}+\mathrm{G}-$ & 2.37 & 2.26 & 1.95 & $\mathrm{C}_{1}$ & 1.4 \\
\hline $\mathrm{TG}+\mathrm{G}+\mathrm{G}+$ & 2.41 & 2.29 & 2.12 & $\mathrm{C}_{1}$ & 1.1 \\
\hline $\mathrm{G}-\mathrm{G}-\mathrm{G}-\mathrm{T}$ & 2.41 & 2.29 & 2.24 & $\mathrm{C}_{1}$ & 0.9 \\
\hline $\mathrm{G}-\mathrm{TG}+\mathrm{T}$ & 2.73 & 2.80 & 2.38 & $\mathrm{C}_{1}$ & 0.7 \\
\hline G-TG- G+ & 2.69 & 2.74 & 2.37 & $\mathrm{C}_{1}$ & 0.7 \\
\hline $\mathrm{TG}-\mathrm{G}+\mathrm{G}+$ & 3.34 & 3.00 & 2.51 & $\mathrm{C}_{1}$ & 0.6 \\
\hline TTG- G+ & 2.65 & 2.77 & 2.47 & $\mathrm{C}_{1}$ & 0.6 \\
\hline TTG- G- & 2.59 & 2.73 & 2.63 & $\mathrm{C}_{1}$ & 0.5 \\
\hline $\mathrm{TG}-\mathrm{G}+\mathrm{G}-$ & 3.28 & 2.95 & 2.68 & $\mathrm{C}_{1}$ & 0.4 \\
\hline $\mathrm{G}-\mathrm{G}-\mathrm{G}+\mathrm{T}$ & 3.32 & 3.00 & 2.89 & $\mathrm{C}_{1}$ & 0.3 \\
\hline $\mathrm{G}+\mathrm{G}+\mathrm{T} \mathrm{G}+$ & 3.10 & 2.99 & 2.91 & $\mathrm{C}_{1}$ & 0.3 \\
\hline G+TT G+ & 3.07 & 3.19 & 3.11 & $\mathrm{C}_{1}$ & 0.2 \\
\hline $\mathrm{G}-\mathrm{G}-\mathrm{G}-\mathrm{G}-$ & 3.90 & 3.70 & 3.39 & $\mathrm{C}_{1}$ & 0.1 \\
\hline $\mathrm{G}-\mathrm{G}-\mathrm{G}+\mathrm{G}-$ & 4.70 & 4.37 & 4.18 & $\mathrm{C}_{1}$ & 0.0 \\
\hline G-TG+ G- & 4.07 & 4.11 & 4.00 & $\mathrm{C}_{1}$ & 0.0 \\
\hline G-TG- G- & 4.05 & 4.12 & 3.97 & $\mathrm{C}_{1}$ & 0.0 \\
\hline
\end{tabular}

Table 1 only few of them are abundant enough to impact vibrational spectrum. Theoretical spectra compared with experimental ones are presented in Figures 7 and 8. Amplified segments of spectra are presented for a better view of details. We have chosen the Cauchy-Gauss product function [19] as the band model, with a constant Cauchy-Gauss ratio and a constant half-width of the bands. Parameters $a_{2}=0.15$ and $a_{4}=0.03$ were used in the product function, resulting in the full-width at half height of $14 \mathrm{~cm}^{-1}$.

There are no proton donor groups in the studied molecule, therefore no association due to hydrogen-bonding is expected. Although a weak proton donor behavior of $\mathrm{C}_{\alpha}-\mathrm{H}$ bonds was demonstrated for $\mathrm{H}$-bonded $n$-propanol [20], the screening by the alkyl groups prevents any interaction with the oxygen atom in a neighboring ether molecule. No evidence was also found in the literature for other types of structure-making forces in liquid ethers, for example, due to dipole-dipole interactions, since the dipole moment of NBEE amounts only to $1.24 \mathrm{D}$ [21]. Thus, the existence of dimers or higher polymers, which could influence vibrational spectra by concentration effects can be safely excluded.

Comparing the results with previous harmonic studies of di-n-propylether [12], it can be noticed that the quality of spectra reproduction is noticeably higher when unscaled B2PLYP/N07D anharmonic calculations are employed. The agreement between the calculated and experimental frequencies as well as the overall shape of the resulting theoretical spectrum is much better than the similar results yielded with harmonic B3LYP calculations even with application of scaling. It is also worth noticing that the agreement with experimental spectra in the lower range $\left(1800-560 \mathrm{~cm}^{-1}\right)$ is still better than in upper range (stretching modes), both in raw frequencies and overall shape of resulting spectra, similarly as for previously studied dipropyl ether [12, 22].

4.6. Identification of Bands in MIR Region. The identification of bands observed in liquid phase spectra, based on a PED analysis and thorough comparison of the experimental $k(v)$ spectrum with modelled IR spectrum are presented in Table 4. The calculated wavenumbers in the table refer to band positions in the modelled spectrum incorporating a number of calculated spectra of conformers. Therefore they differ from the calculated wavenumbers for individual conformers.

As stated before, the quality of experimental spectra reproduction is lower in the $\mathrm{C}-\mathrm{H}$ stretching region-in terms of band positions and relative intensities as well, resulting in overall different spectrum shape. Moreover, in this region, the differences of modes frequencies between conformers are highest; therefore we can see a significant level of band overlapping here. Nevertheless main bands can still be clearly 
TABLE 2: Definitions of internal coordinates for NBEE molecule.

\begin{tabular}{|c|c|c|}
\hline Coord. & Definition & Description \\
\hline 1 & O1-C2 stretch. & C-O stretch. \\
\hline 2 & O1-C5 stretch. & $\mathrm{C}-\mathrm{O}$ stretch. \\
\hline 3 & C2-H3 stretch. & $\mathrm{C}\left(\mathrm{H}_{2}\right)-\mathrm{H}$ stretch. \\
\hline 4 & C2-H4 stretch. & $\mathrm{C}\left(\mathrm{H}_{2}\right)-\mathrm{H}$ stretch. \\
\hline 5 & C2-C18 stretch. & C-C stretch. \\
\hline 6 & C5-H6 stretch. & $\mathrm{C}\left(\mathrm{H}_{2}\right)-\mathrm{H}$ stretch. \\
\hline 7 & C5-H7 stretch. & $\mathrm{C}\left(\mathrm{H}_{2}\right)-\mathrm{H}$ stretch. \\
\hline 8 & C5-C8 stretch. & C-C stretch. \\
\hline 9 & C8-H9 stretch. & $\mathrm{C}\left(\mathrm{H}_{2}\right)-\mathrm{H}$ stretch. \\
\hline 10 & C8-H10 stretch. & $\mathrm{C}\left(\mathrm{H}_{2}\right)-\mathrm{H}$ stretch. \\
\hline 11 & C8-C11 stretch. & C-C stretch. \\
\hline 12 & C11-H12 stretch. & $\mathrm{C}\left(\mathrm{H}_{2}\right)-\mathrm{H}$ stretch. \\
\hline 13 & C11-H13 stretch. & $\mathrm{C}\left(\mathrm{H}_{2}\right)-\mathrm{H}$ stretch. \\
\hline 14 & C11-C14 stretch. & C-C stretch. \\
\hline 15 & C14-H15 stretch. & $\mathrm{C}\left(\mathrm{H}_{3}\right)-\mathrm{H}$ stretch. \\
\hline 16 & C14-H16 stretch. & $\mathrm{C}\left(\mathrm{H}_{3}\right)-\mathrm{H}$ stretch. \\
\hline 17 & C14-H17 stretch. & $\mathrm{C}\left(\mathrm{H}_{3}\right)-\mathrm{H}$ stretch. \\
\hline 18 & C18-H19 stretch. & $\mathrm{C}\left(\mathrm{H}_{3}\right)-\mathrm{H}$ stretch. \\
\hline 19 & C18-H20 stretch. & $\mathrm{C}\left(\mathrm{H}_{3}\right)-\mathrm{H}$ stretch. \\
\hline 20 & C18-H21 stretch. & $\mathrm{C}\left(\mathrm{H}_{3}\right)-\mathrm{H}$ stretch. \\
\hline 21 & $\mathrm{C}(18) \mathrm{H}_{3}$ sym. def. & $\mathrm{CH}_{3}$ sym. def. \\
\hline 22 & $\mathrm{C}(18) \mathrm{H}_{3}$ asym. def. & $\mathrm{CH}_{3}$ asym. def. \\
\hline 23 & $\mathrm{C}(18) \mathrm{H}_{3}$ asym. def.' & $\mathrm{CH}_{3}$ asym. def.' \\
\hline 24 & $\mathrm{C}(18) \mathrm{H}_{3}$ rock. & $\mathrm{CH}_{3}$ rock. \\
\hline 25 & $\mathrm{CH}_{3}$ rock.' & $\mathrm{CH}_{3}$ rock.' \\
\hline 26 & $\mathrm{C}(14) \mathrm{H}_{3}$ sym. def. & $\mathrm{CH}_{3}$ sym. def. \\
\hline 27 & $\mathrm{C}(14) \mathrm{H}_{3}$ asym. def. & $\mathrm{CH}_{3}$ asym. def. \\
\hline 28 & $\mathrm{C}(14) \mathrm{H}_{3}$ asym. def.' & $\mathrm{CH}_{3}$ asym. def.' \\
\hline 29 & $\mathrm{C}(14) \mathrm{H}_{3}$ rock. & $\mathrm{CH}_{3}$ rock. \\
\hline 30 & $\mathrm{C}(14) \mathrm{H}_{3}$ rock.' & $\mathrm{CH}_{3}$ rock.' \\
\hline 31 & $\mathrm{C}(2) \mathrm{H}_{2}$ sciss. & $\mathrm{CH}_{2}$ sciss. \\
\hline 32 & $\mathrm{C}(2) \mathrm{H}_{2}$ sciss. & $\mathrm{CH}_{2}$ sciss. \\
\hline 33 & $\mathrm{C}(2) \mathrm{H}_{2}$ rock. & $\mathrm{CH}_{2}$ rock. \\
\hline 34 & $\mathrm{C}(2) \mathrm{H}_{2}$ wagg. & $\mathrm{CH}_{2}$ wagg. \\
\hline 35 & $\mathrm{C}(2) \mathrm{H}_{2}$ twist. & $\mathrm{CH}_{2}$ twist. \\
\hline 36 & $\mathrm{C}(5) \mathrm{H}_{2}$ sciss. & $\mathrm{CH}_{2}$ sciss. \\
\hline 37 & $\mathrm{C}(5) \mathrm{H}_{2}$ sciss. & $\mathrm{CH}_{2}$ sciss. \\
\hline 38 & $\mathrm{C}(5) \mathrm{H}_{2}$ rock. & $\mathrm{CH}_{2}$ rock. \\
\hline 39 & $\mathrm{C}(5) \mathrm{H}_{2}$ wagg. & $\mathrm{CH}_{2}$ wagg. \\
\hline 40 & $\mathrm{C}(5) \mathrm{H}_{2}$ twist. & $\mathrm{CH}_{2}$ twist. \\
\hline 41 & $\mathrm{C}(8) \mathrm{H}_{2}$ sciss. & $\mathrm{CH}_{2}$ sciss. \\
\hline 42 & $\mathrm{C}(8) \mathrm{H}_{2}$ sciss. & $\mathrm{CH}_{2}$ sciss. \\
\hline 43 & $\mathrm{C}(8) \mathrm{H}_{2}$ rock. & $\mathrm{CH}_{2}$ rock. \\
\hline 44 & $\mathrm{C}(8) \mathrm{H}_{2}$ wagg. & $\mathrm{CH}_{2}$ wagg. \\
\hline 45 & $\mathrm{C}(8) \mathrm{H}_{2}$ twist. & $\mathrm{CH}_{2}$ twist. \\
\hline 46 & $\mathrm{C}(11) \mathrm{H}_{2}$ sciss. & $\mathrm{CH}_{2}$ sciss. \\
\hline 47 & $\mathrm{C}(11) \mathrm{H}_{2}$ sciss. & $\mathrm{CH}_{2}$ sciss. \\
\hline 48 & $\mathrm{C}(11) \mathrm{H}_{2}$ rock. & $\mathrm{CH}_{2}$ rock. \\
\hline 49 & $\mathrm{C}(11) \mathrm{H}_{2}$ wagg. & $\mathrm{CH}_{2}$ wagg. \\
\hline 50 & $\mathrm{C}(11) \mathrm{H}_{2}$ twist. & $\mathrm{CH}_{2}$ twist. \\
\hline
\end{tabular}

TABLE 2: Continued.

\begin{tabular}{lcc}
\hline Coord. & Definition & Description \\
\hline 51 & C2-C8 tors. & C-C tors. \\
52 & C2-O1 tors & C-O tors \\
53 & C5-O1 tors & C-O tors \\
54 & C5-C8 tors. & C-C tors. \\
55 & C8-C11 tors. & C-C tors. \\
56 & C11-C14 tors. & C-C tors. \\
57 & C2-O1-C5 bend. & C-O-C bend. \\
\hline
\end{tabular}

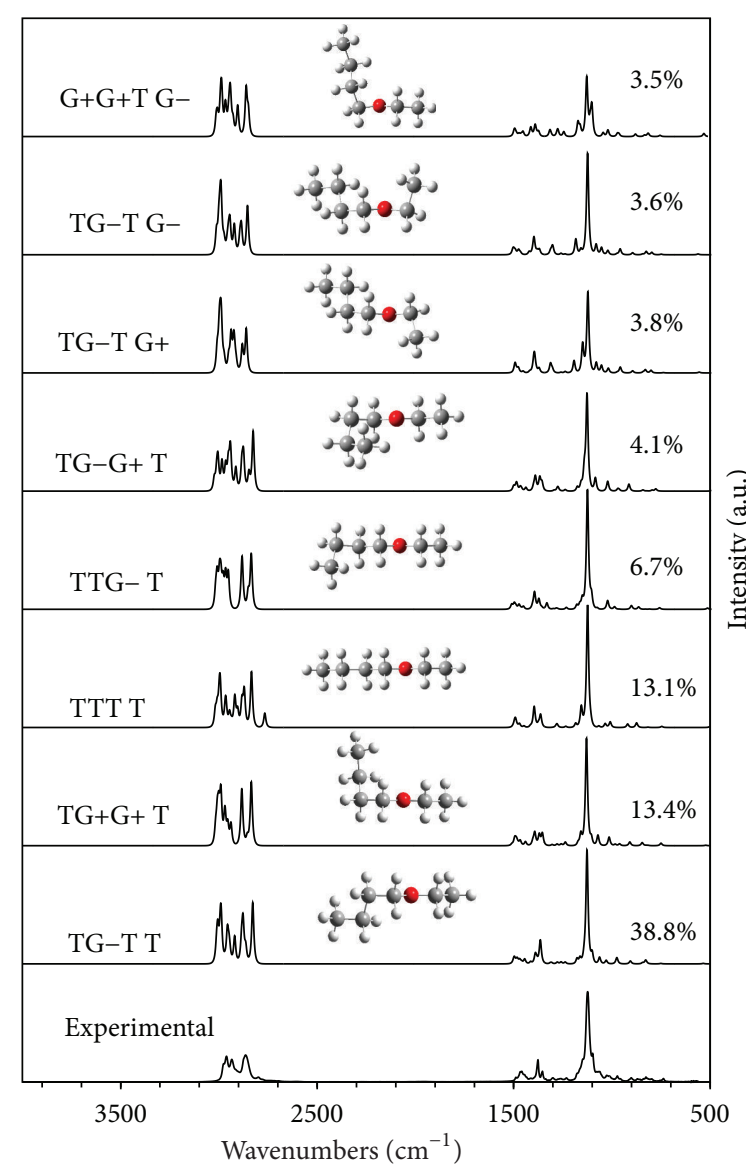

FIGURE 6: Experimental $k(v)$ spectrum of liquid NBEE and simulated B2PLYP/N07D vibrational spectra of most abundant NBEE conformers (abundance larger than 3\%).

identified. The $\mathrm{CH}_{3}$ stretching asymmetric and $\mathrm{CH}_{2}$ stretching asymmetric, as well as stretching symmetric modes for those groups, have been identified (Table 4). Unexpectedly, the $2864.4 \mathrm{~cm}^{-1}$ band was found to be a $\mathrm{CH}_{2}$ (ethyl chain) stretching asymmetric band, strongly shifted towards lower wavenumber.

Aliphatic ethers and thioethers exhibit several weak bands at $2800-2700 \mathrm{~cm}^{-1}$ range which are absent in alkanes. In the NBEE spectrum, we can observe such bands too, with the most prominent one at $2799 \mathrm{~cm}^{-1}$ wavenumber. We are not exactly sure about the origin of those bands; however, our 
TABLE 3: PED table for the major conformer (“TG-T T") of NBEE.

\begin{tabular}{|c|c|c|c|c|c|c|c|c|c|c|c|c|c|c|c|c|}
\hline \multicolumn{3}{|c|}{ Calculated frequency $\left(\mathrm{cm}^{-1}\right)$} & \multirow{2}{*}{ Mode } & \multirow{2}{*}{$(\%)$} & \multirow{2}{*}{ Mode } & \multirow{2}{*}{$(\%)$} & \multirow{2}{*}{ Mode } & \multirow{2}{*}{$(\%)$} & \multirow{2}{*}{ Mode } & \multirow{2}{*}{$(\%)$} & \multirow{2}{*}{ Mode } & \multirow{2}{*}{$(\%)$} & \multirow{2}{*}{ Mode } & \multirow{2}{*}{$(\%)$} & \multirow{2}{*}{ Mode } & \multirow{2}{*}{ (\%) } \\
\hline & Harmonic & Anharmonic & & & & & & & & & & & & & & \\
\hline 1 & 72.2 & 59.4 & 52 & (48) & 54 & (22) & 53 & (10) & & & & & & & & \\
\hline 2 & 73.9 & 80.2 & 55 & (59) & 54 & (13) & 52 & $(6)$ & 37 & (6) & & & & & & \\
\hline 3 & 78.3 & 68.1 & 53 & $(71)$ & 42 & $(10)$ & & & & & & & & & & \\
\hline 4 & 140.5 & 134.1 & 52 & $(35)$ & -54 & $(28)$ & -53 & (7) & 37 & (6) & 42 & (6) & & & & \\
\hline 5 & 210.4 & 210.8 & 57 & (29) & -32 & $(20)$ & -54 & $(15)$ & -37 & (13) & 55 & (9) & & & & \\
\hline 6 & 244.1 & 229.1 & 51 & (69) & -56 & (11) & -42 & $(6)$ & & & & & & & & \\
\hline 7 & 249.9 & 245.2 & 56 & (68) & 51 & (16) & -54 & (6) & & & & & & & & \\
\hline 8 & 272.3 & 273.9 & 47 & (38) & -42 & (33) & -54 & (6) & 53 & (5) & & & & & & \\
\hline 9 & 329.5 & 327.9 & 47 & (27) & 37 & (18) & 57 & (12) & 8 & (10) & 42 & (9) & & & & \\
\hline 10 & 442.7 & 440.6 & 32 & (50) & -37 & (12) & -24 & (11) & 57 & (11) & & & & & & \\
\hline 11 & 540.1 & 535.0 & 37 & (23) & -42 & (18) & -47 & (11) & 57 & (9) & 43 & (8) & & & & \\
\hline 12 & 747.7 & 750.9 & 48 & (45) & 43 & (26) & 30 & (10) & & & & & & & & \\
\hline 13 & 830.9 & 823.1 & 25 & (41) & -33 & (37) & -35 & (13) & & & & & & & & \\
\hline 14 & 842.1 & 828.1 & 30 & (20) & -43 & (17) & 48 & (10) & 2 & (9) & 1 & (8) & -50 & (7) & 24 & (6) \\
\hline 15 & 859.5 & 843.9 & 11 & (32) & 29 & (16) & 14 & (9) & -38 & (8) & 8 & (8) & & & & \\
\hline 16 & 922.2 & 906.0 & 24 & (24) & 1 & (15) & -30 & (11) & 5 & (10) & 50 & (8) & 43 & (8) & -37 & (6) \\
\hline 17 & 987.9 & 972.6 & 38 & (30) & 29 & (28) & -44 & (9) & -49 & $(8)$ & -40 & (6) & & & & \\
\hline 18 & 995.3 & 975.1 & 5 & (16) & -2 & (15) & -8 & (14) & 45 & (13) & 30 & (10) & -50 & (9) & & \\
\hline 19 & 1054.7 & 1028.9 & 14 & (52) & -5 & (11) & 2 & (9) & -8 & $(8)$ & -11 & $(7)$ & & & & \\
\hline 20 & 1087.1 & 1062.8 & 5 & (23) & 8 & (17) & -1 & (15) & 14 & (13) & -11 & (12) & & & & \\
\hline 21 & 1129.6 & 1100.4 & 24 & (19) & -5 & (14) & -11 & (13) & 8 & (11) & 32 & $(8)$ & -2 & (6) & 29 & (6) \\
\hline 22 & 1165.1 & 1126.7 & 1 & (41) & -2 & (40) & & & & & & & & & & \\
\hline 23 & 1167.1 & 1141.0 & 38 & (20) & -29 & (16) & 11 & (13) & 24 & (7) & -42 & (7) & -47 & (6) & & \\
\hline 24 & 1190.0 & 1162.4 & 33 & (18) & 25 & (11) & -43 & (9) & 24 & (8) & 48 & (7) & -1 & (6) & -30 & (5) \\
\hline 25 & 1203.9 & 1177.6 & 33 & (30) & 25 & (16) & 38 & (11) & 43 & (8) & -48 & (6) & & & & \\
\hline 26 & 1268.7 & 1237.6 & 40 & (41) & 45 & (14) & -30 & (11) & 48 & (8) & -50 & (8) & & & & \\
\hline 27 & 1293.1 & 1258.7 & 40 & (24) & -45 & (21) & 50 & (14) & 44 & (9) & 30 & (6) & & & & \\
\hline 28 & 1309.9 & 1277.9 & 35 & (84) & 25 & (10) & & & & & & & & & & \\
\hline 29 & 1339.9 & 1306.7 & 50 & (40) & -49 & (16) & 45 & (16) & -44 & (11) & 40 & (6) & & & & \\
\hline 30 & 1345.9 & 1313.2 & 49 & (30) & 45 & (16) & 44 & (16) & -40 & (13) & 50 & (12) & & & & \\
\hline 31 & 1395.6 & 1364.2 & 34 & (46) & 39 & (31) & 21 & (13) & & & & & & & & \\
\hline 32 & 1419.5 & 1382.6 & 44 & (26) & -21 & (23) & -49 & $(21)$ & 39 & (9) & 11 & (9) & & & & \\
\hline 33 & 1424.1 & 1389.6 & 21 & $(42)$ & 44 & (14) & -39 & (12) & -49 & (8) & -8 & (7) & & & & \\
\hline 34 & 1430.5 & 1413.7 & 26 & $(82)$ & 14 & (10) & & & & & & & & & & \\
\hline 35 & 1462.3 & 1443.9 & 34 & (35) & -39 & (29) & -21 & (9) & -5 & (9) & -8 & (5) & & & & \\
\hline 36 & 1491.4 & 1459.7 & 41 & (88) & 46 & $(5)$ & & & & & & & & & & \\
\hline 37 & 1498.7 & 1469.1 & 22 & (69) & -23 & (22) & -25 & (8) & & & & & & & & \\
\hline 38 & 1510.0 & 1479.3 & 46 & (48) & -28 & (36) & -27 & (7) & & & & & & & & \\
\hline 39 & 1515.1 & 1498.0 & 27 & (75) & -28 & (16) & -30 & (8) & & & & & & & & \\
\hline 40 & 1516.5 & 1484.8 & 23 & (55) & 22 & (18) & -31 & (14) & -24 & (6) & & & & & & \\
\hline 41 & 1523.3 & 1495.1 & 46 & (45) & 28 & (33) & 27 & (7) & & & & & & & & \\
\hline 42 & 1526.4 & 1481.3 & 36 & (64) & -31 & (21) & & & & & & & & & & \\
\hline 43 & 1544.4 & 1502.1 & 31 & (61) & 36 & (25) & & & & & & & & & & \\
\hline 44 & 2985.8 & 2863.6 & 6 & (54) & 7 & (28) & -4 & (8) & -3 & (8) & & & & & & \\
\hline 45 & 2999.3 & 2828.0 & 4 & (42) & 3 & (41) & 6 & (12) & & & & & & & & \\
\hline 46 & 3022.6 & 2877.3 & 7 & (59) & -6 & (31) & & & & & & & & & & \\
\hline 47 & 3029.5 & 2884.4 & 3 & (48) & -4 & (46) & & & & & & & & & & \\
\hline 48 & 3050.8 & 2944.8 & 10 & (34) & 13 & (28) & 9 & (25) & 12 & (5) & & & & & & \\
\hline 49 & 3055.5 & 2919.9 & 13 & (57) & -10 & (24) & 12 & $(6)$ & & & & & & & & \\
\hline 50 & 3058.4 & 2956.9 & 17 & (36) & 16 & (29) & 15 & (24) & 10 & (7) & & & & & & \\
\hline
\end{tabular}


TABLE 3: Continued.

\begin{tabular}{|c|c|c|c|c|c|c|c|c|c|c|c|c|c|c|c|c|}
\hline \multicolumn{3}{|c|}{ Calculated frequency $\left(\mathrm{cm}^{-1}\right)$} & \multirow{2}{*}{ Mode } & \multirow{2}{*}{$(\%)$} & \multirow{2}{*}{ Mode } & \multirow{2}{*}{$(\%)$} & \multirow{2}{*}{ Mode } & \multirow{2}{*}{$(\%)$} & \multirow{2}{*}{ Mode } & \multirow{2}{*}{$(\%)$} & \multirow{2}{*}{ Mode } & \multirow{2}{*}{$(\%)$} & \multirow{2}{*}{ Mode } & \multirow{2}{*}{$(\%)$} & \multirow{2}{*}{ Mode } & \multirow{2}{*}{$(\%)$} \\
\hline & Harmonic & Anharmonic & & & & & & & & & & & & & & \\
\hline 51 & 3074.6 & 3006.3 & 18 & $(40)$ & 20 & $(30)$ & 19 & (30) & & & & & & & & \\
\hline 52 & 3094.0 & 2949.3 & 9 & (56) & -10 & $(25)$ & -12 & (17) & & & & & & & & \\
\hline 53 & 3113.3 & 2969.0 & 12 & (43) & 17 & $(20)$ & -16 & (15) & 9 & $(7)$ & -13 & (7) & -10 & $(6)$ & & \\
\hline 54 & 3134.3 & 2988.8 & 16 & (35) & -17 & $(29)$ & 12 & (25) & -13 & (7) & & & & & & \\
\hline 55 & 3136.7 & 2993.0 & 15 & (72) & -16 & (14) & -17 & (11) & & & & & & & & \\
\hline 56 & 3152.2 & 3005.6 & 18 & (60) & -20 & (21) & -19 & (19) & & & & & & & & \\
\hline 57 & 3160.9 & 3011.5 & 19 & (51) & -20 & (48) & & & & & & & & & & \\
\hline
\end{tabular}

TABLE 4: MIR and Raman band identification for liquid NBEE.

\begin{tabular}{|c|c|c|c|c|}
\hline \multicolumn{3}{|c|}{$v_{\exp }\left(\mathrm{cm}^{-1}\right)$} & \multirow{2}{*}{$\begin{array}{l}v_{\text {calc }} \\
\left(\mathrm{cm}^{-1}\right)\end{array}$} & \multirow{2}{*}{ Vibrational modes contributions } \\
\hline & IR & Raman & & \\
\hline 1 & 2975.5 & 2975.7 & 3003 & $\mathrm{CH}_{3}$ str. asym. \\
\hline 2 & 2961.5 & 2962.5 & 2991 & $\mathrm{CH}_{3}$ str. asym., $\mathrm{CH}_{2}$ str. asym. \\
\hline 3 & 2934.6 & 2934.1 & 2955 & $\mathrm{CH}_{3}$ str. asym., $\mathrm{CH}_{2}$ str. asym. \\
\hline 4 & $\sim 2913$ & 2913.4 & 2919 & $\mathrm{CH}_{2}$ str. sym. \\
\hline 5 & $\sim 2874$ & 2873.4 & 2884 & $\mathrm{CH}_{2}$ str. asym. \\
\hline 6 & 2864.4 & 2868.9 & 2861 & $\mathrm{CH}_{2}$ str. asym. \\
\hline 7 & 2799.3 & 2799.2 & - & - \\
\hline 8 & 1488.4 & 1487.0 & 1495 & $\mathrm{CH}_{2}$ sciss., $\mathrm{CH}_{3}$ asym. def.' \\
\hline 9 & 1466.0 & - & 1469 & $\mathrm{CH}_{3}$ asym. def. \\
\hline 9 & 1458.7 & $\sim 1457$ & - & - \\
\hline 10 & 1412.5 & - & 1414 & $\mathrm{CH}_{3}$ sym. def. \\
\hline 11 & 1376.3 & - & 1390 & $\mathrm{CH}_{3}$ sym. def. \\
\hline 12 & 1333.5 & - & 1364 & $\mathrm{CH}_{2}$ wagg. \\
\hline 13 & 1300.2 & 1299.0 & 1307 & $\mathrm{CH}_{2}$ twist. \\
\hline 14 & 1262.2 & $\sim 1257$ & 1259 & $\mathrm{CH}_{2}$ twist. \\
\hline 15 & 1231.4 & 1230.8 & 1237 & $\mathrm{CH}_{2}$ twist. \\
\hline 16 & 1123.2 & 1119.9 & 1126 & $\mathrm{C}-\mathrm{O}$ stretch. \\
\hline 17 & 1099.0 & 1099.2 & 1100 & $\mathrm{CH}_{3}$ rock., $\mathrm{C}-\mathrm{C}$ stretch. \\
\hline 18 & 1067.9 & $\sim 1066$ & 1062 & C-C stretch. \\
\hline 19 & 1026.5 & 1027.7 & 1028 & C-C stretch. \\
\hline 20 & 972.4 & $\sim 972$ & 975 & C-C stretch. \\
\hline 21 & 901.5 & 901.9 & 905 & $\mathrm{CH}_{3}$ rock. \\
\hline 22 & 870.7 & - & 871 & $\mathrm{C}-\mathrm{C}$ stretch., $\mathrm{CH}_{3}$ rock. \\
\hline 23 & 826.7 & $\sim 828$ & 828 & $\mathrm{CH}_{3}$ rock.', $\mathrm{CH}_{2}$ rock. \\
\hline
\end{tabular}

calculations suggest at least a contribution of fundamental bands. According to collected data, a few of investigated NBEE conformers contain a significantly shifted symmetric stretching band of $\alpha-\mathrm{CH}_{2}$ groups, including the third most abundant "TTT T" conformer with the band at a calculated frequency $2766 \mathrm{~cm}^{-1}$. Such a high shift of $\alpha-\mathrm{CH}_{2}$ symmetric stretching band has been observed for all previously studied aliphatic ethers.

The lower MIR region is dominated by $\mathrm{CH}_{3}$ asymmetric deformation, $\mathrm{CH}_{2}$ scissoring, and $\mathrm{CH}_{2}$ wagging modes (in the $1500-1300 \mathrm{~cm}^{-1}$ range), and by $\mathrm{CH}_{2}$ twisting, $\mathrm{C}-\mathrm{O}$

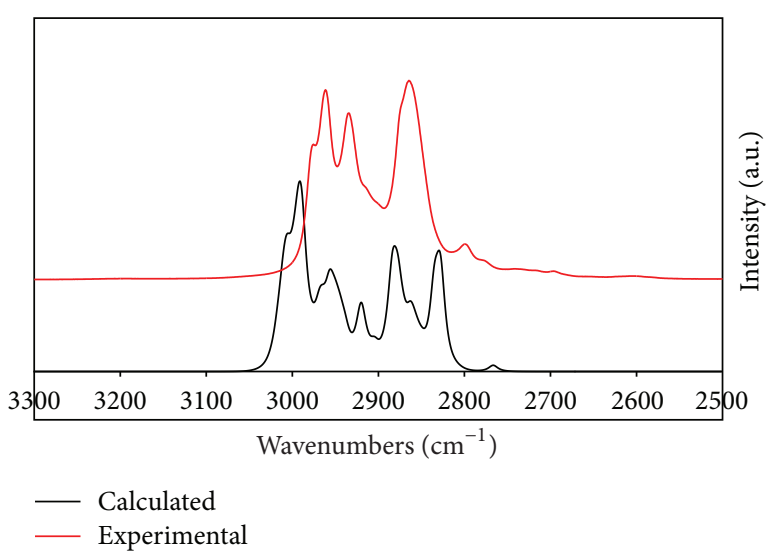

FIgURE 7: Calculated and experimental spectra of NBEE in the upper MIR range $\left(3300-2500 \mathrm{~cm}^{-1}\right)$.

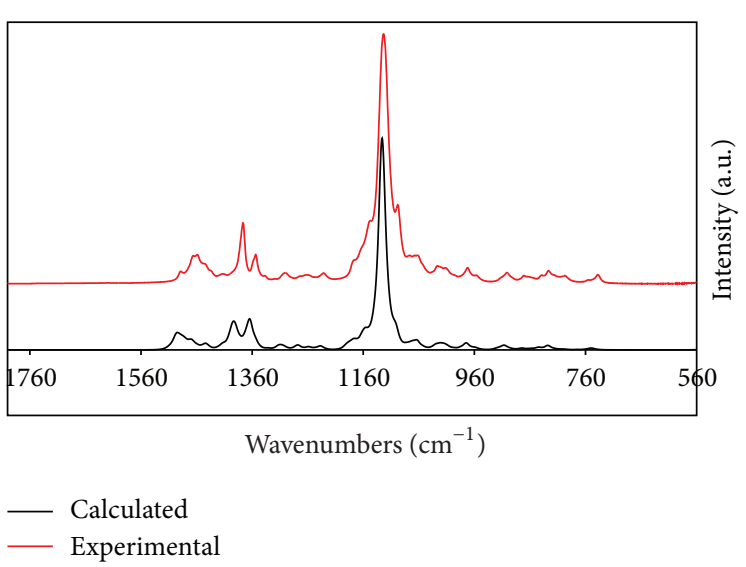

FIGURE 8: Calculated and experimental spectra of NBEE in the lower MIR range $\left(1800-560 \mathrm{~cm}^{-1}\right)$.

stretching, and C-C stretching modes (in the 1300-1000 $\mathrm{cm}^{-1}$ range). The broadening of $\mathrm{C}-\mathrm{O}$ stretching band due to overlapping bands of conformers can be easily observed. Due to this effect also the relative intensity of $\mathrm{C}-\mathrm{O}$ band is slightly lower than expected; it is; however, still the most intense band in the spectrum. Also a considerable contribution of $\mathrm{C}-\mathrm{C}$ stretching bands into the broadened $\mathrm{C}-\mathrm{O}$ stretching band in studied spectra is probable. 
Summarizing, the influence of oxygen atom can be pointed out in the spectrum of investigated ether. According to the analyzed data, both $\mathrm{CH}_{2}(-\mathrm{O})$ groups in NBEE differ from other methylene groups. The presence of multiple relevant conformers to band broadening and overlapping which is mostly noticeable in case of $\mathrm{C}-\mathrm{H}$ stretching range and $\mathrm{C}-\mathrm{O}$ stretching band.

\section{Summary}

Basing on IR studies including thin-film MIR transmission recordings, the spectrum of the complex refractive index for liquid $n$-butyethylether was determined in a broad spectral range. A detailed conformer population analysis and anharmonic vibrational analysis was performed on a B2PLYP/N07D level of theory. The observed spectrum of neat liquid was accurately reproduced, and a successful identification of numerous MIR bands of studied liquid was carried out.

\section{Acknowledgment}

Calculations have been partially carried out in Wroclaw Centre for Networking and Supercomputing (http://www .wcss.wroc.pl), Grant no. 20207.

\section{References}

[1] D. Montaño, I. Gascón, B. Schmid, J. Gmehling, and C. Lafuente, "Experimental and predicted properties of the binary mixtures containing an isomeric chlorobutane and butyl ethyl ether," The Journal of Chemical Thermodynamics, vol. 51, pp. 150-158, 2012.

[2] B. J. Deelman, M. Booij, A. Meetsma, J. H. Teuben, H. Kooijman, and A. L. Spek, "Activation of ethers and sulfides by organolanthanide hydrides. Molecular structures of $\left(\mathrm{Cp}^{*}{ }_{2} \mathrm{Y}\right)_{2}\left(\mu-\mathrm{OCH}_{2} \mathrm{CH}_{2} \mathrm{O}\right)(\mathrm{THF})_{2}$ and $\left(\mathrm{Cp}_{2}{ }_{2} \mathrm{Ce}\right)_{2}(\mu-$ $\mathrm{O})(\mathrm{THF})_{2}$," Organometallics, vol. 14 , no. 5, pp. 2306-2317, 1995.

[3] A. Hull, I. Golubkov, B. Kronberg, and J. Van Stam, "Alternative fuel for a standard diesel engine," International Journal of Engine Research, vol. 7, no. 1, pp. 51-63, 2006.

[4] P. J. Bennett and J. A. Kerr, "Kinetics of the reactions of hydroxyl radicals with aliphatic ethers studied under simulated atmospheric conditions," Journal of Atmospheric Chemistry, vol. 8, no. 1, pp. 87-94, 1989.

[5] D. Johnson and J. M. Andino, "Laboratory studies of the $\cdot \mathrm{OH}-$ initiated photooxidation of ethyl- $n$-butyl ether and di- $n$-butyl ether," International Journal of Chemical Kinetics, vol. 33, no. 5, pp. 328-341, 2001.

[6] M. Kaykhaii and M. R. Mirbaloochzahi, "Direct screening of ground water samples for fuel oxygenates by headspace liquid phase microextraction-gas chromatography," Environmental Monitoring and Assessment, vol. 147, no. 1-3, pp. 211-222, 2008.

[7] J. E. Bertie, Handbook of Vibrational Spectroscopy, vol. 1, John Wiley and Sons, Chichester, UK, 2002.

[8] J. P. Hawranek, P. Neelakantan, R. P. Young, and R. N. Jones, "The control of errors in i.r. spectrophotometry-III.
Transmission measurements using thin cells," Spectrochimica Acta, vol. 32, no. 1, pp. 75-84, 1976.

[9] J. P. Hawranek, P. Neelakantan, R. P. Young, and R. N. Jones, "The control of errors in i.r. spectrophotometry-IV. Corrections for dispersion distortion and the evaluation of both optical constants," Spectrochimica Acta, vol. 32, no. 1, pp. 85-98, 1976.

[10] W. Wrzeszcz, A. S. Muszyński, and J. P. Hawranek, "Analysis of IR thin-film transmission spectra of liquid tri- $n$-propylamine," Computers and Chemistry, vol. 22, no. 1, pp. 101-111, 1998.

[11] J. P. Hawranek and A. S. Muszyński, "On the determination of optical constants of liquids in the infrared region," Computers and Chemistry, vol. 22, no. 1, pp. 95-100, 1998.

[12] A. S. Muszyński, K. B. Beć, W. Wrzeszcz, N. Michniewicz, A. Mojak, and J. P. Hawranek, "Vibrational spectra of liquid di- $n$ propylether," Journal of Molecular Structure, vol. 975, no. 1-3, pp. 205-210, 2010.

[13] B. I. Łydżba-Kopczyńska, K. B. Beć, J. Tomczak, and J. P. Hawranek, "Optical constants of liquid pyrrole in the infrared," Journal of Molecular Liquids, vol. 172, pp. 34-40, 2012.

[14] M. Biczysko, P. Panek, G. Scalmani, J. Bloino, and V. Barone, "Harmonic and anharmonic vibrational frequency calculations with the double-hybrid B2PLYP method: analytic second derivatives and benchmark studies," Journal of Chemical Theory and Computation, vol. 6, no. 7, pp. 2115-2125, 2010.

[15] K. B. Beć and J. P. Hawranek, "Vibrational analysis of liquid $n$ butylmethylether," Vibrational Spectroscopy. In press.

[16] M. J. Frisch, G. W. Trucks, H. B. Schlegel et al., Gaussian 09, Revision A. 02, Gaussian, Wallingford, Conn, USA, 2009.

[17] J. M. L. Martin and C. Van Alsenoy, GAR2PED, University of Antwerp, 1995.

[18] P. Pulay, G. Fogarasi, F. Pang, and J. E. Boggs, "Systematic $\mathrm{ab}$ initio gradient calculation of molecular geometries, force constants, and dipole moment derivatives," Journal of the American Chemical Society, vol. 101, no. 10, pp. 2550-2560, 1979.

[19] J. P. Hawranek, "On the numerical description of asymmetric absorption bands," Acta Physica Polonica B, vol. 40, p. 811, 1971.

[20] S. Jarmelo, N. Maiti, V. Anderson, P. R. Carey, and R. Fausto, " $\mathrm{C}_{\alpha}-\mathrm{H}$ bond-stretching frequency in alcohols as a probe of hydrogen-bonding strength: a combined vibrational spectroscopic and theoretical study of $n$-[1-D]propanol," Journal of Physical Chemistry A, vol. 109, no. 10, pp. 2069-2077, 2005.

[21] J. A. Riddick, W. B. Bunger, and T. K. Sakano, Organic Solvents: Physical Properties and Methods of Purification, John Wiley and Sons, New York, NY, USA, 4th edition, 1986.

[22] K. B. Beć, A. S. Muszyński, N. Michniewicz, W. Wrzeszcz, A. Kotynia, and J. P. Hawranek, "Vibrational spectra of liquid diiso-propylether," Vibrational Spectroscopy, vol. 55, no. 1, pp. 44-48, 2011. 

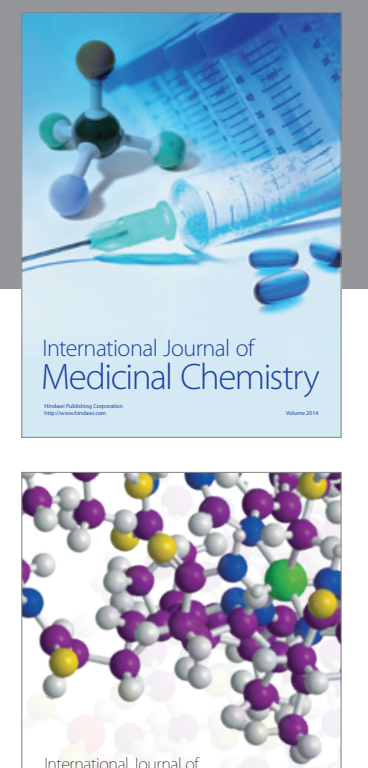

\section{Carbohydrate} Chemistry

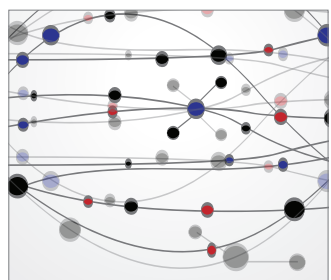

The Scientific World Journal
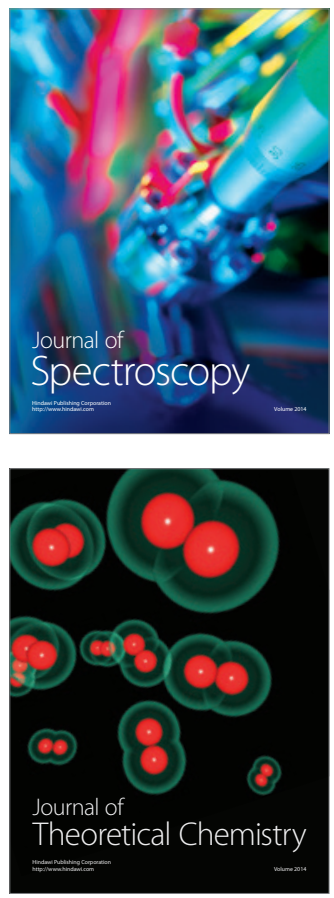
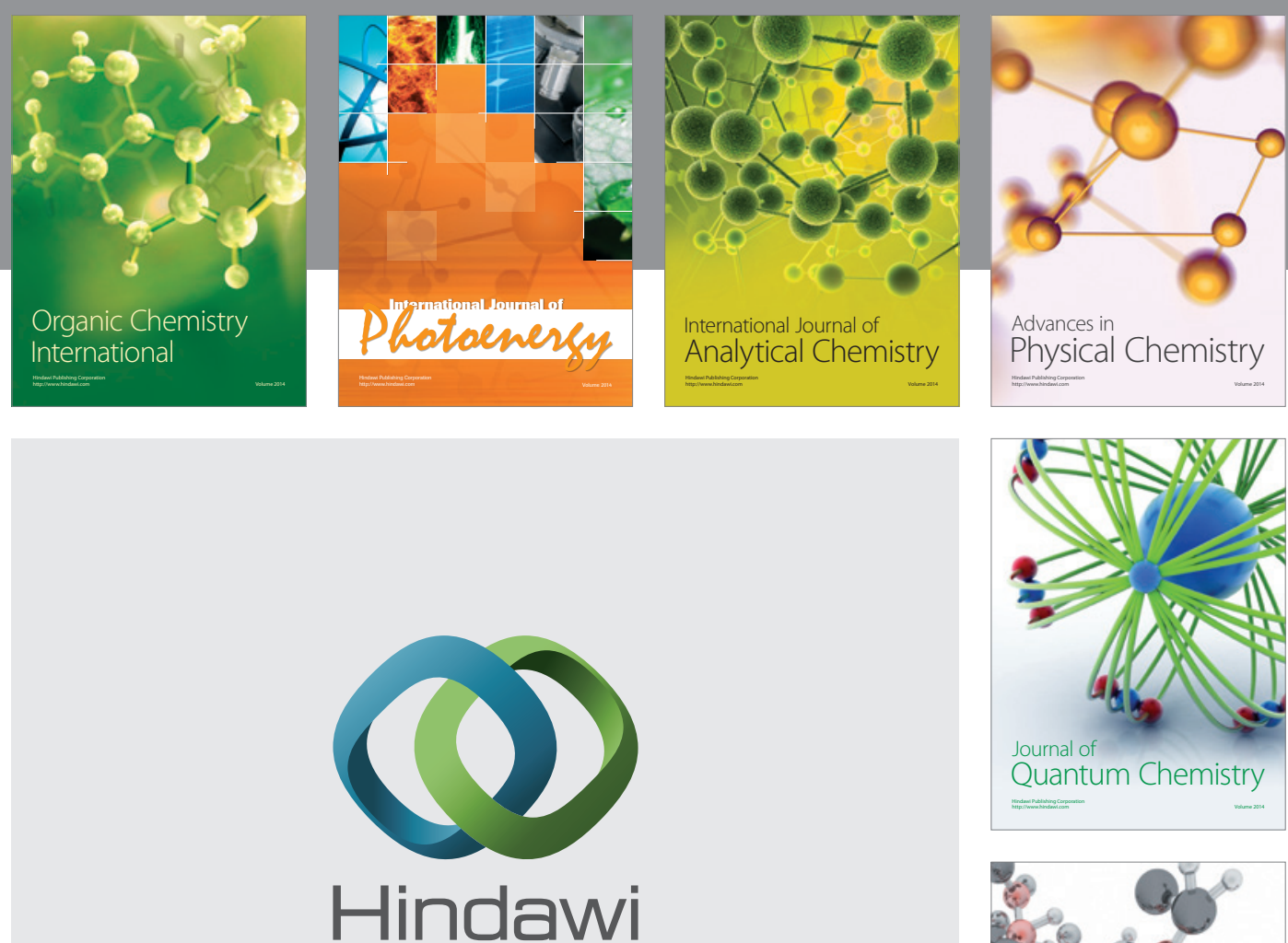

Submit your manuscripts at

http://www.hindawi.com

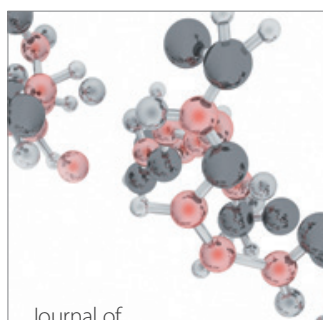

Analytical Methods

in Chemistry

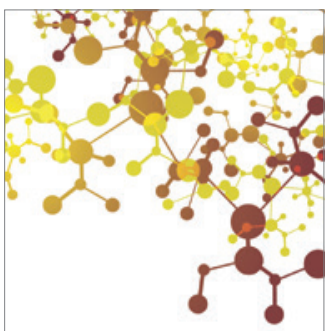

Journal of

Applied Chemistry

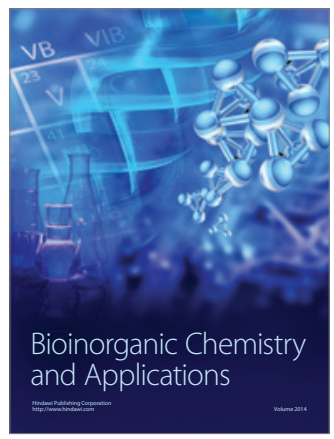

Inorganic Chemistry
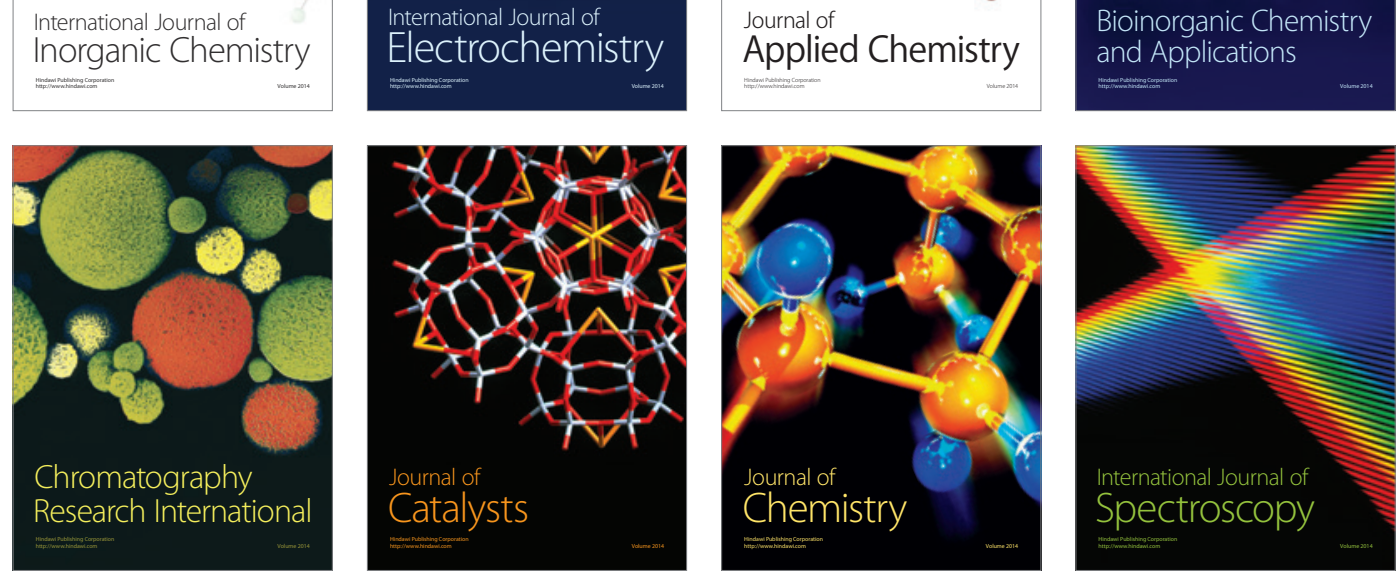